\title{
GESTIÓN TURÍSTICA DEL PATRIMONIO CULTURAL: EL CASO DE ETNIA MATLATZINCA EN EL ESTADO DE MÉXICO
}

\section{TOURISM MANAGEMENT OF CULTURAL HERITAGE: CASE OF THE MATLATZINCA ETHNIC GROUP IN THE STATE OF MEXICO}

\section{González-Domínguez, I. ${ }^{1}$; Thomé-Ortíz, $\mathrm{H}^{2^{*}}$}

\begin{abstract}
${ }^{1}$ Universidad Autónoma del Estado de México, Facultad de Turismo y Gastronomía. Cerro de Coatepec S/N, Ciudad Universitaria, Toluca, Estado de México, México. Universidad Autónoma del Estado de México, Instituto de Ciencias Agropecuarias y Rurales. El Cerrillo Piedras Blancas, Toluca, Estado de México, México.
\end{abstract}

*Autor para correspondencia: humbertothomeahotmail.com

\begin{abstract}
This paper describes the implementation of a tourism management strategy for endogenous resources in the village called San Francisco Oxtotilpan, in the state of Mexico. It is a Matlatzinca community that has natural resources framed in a vast cultural richness, which has been maintained through the preservation of customs and traditions. A case study was carried out, with a focus on the action research methodology, with the purpose of identifying participatory heritage management processes in the implementation of rural tourism strategies. The application of a collaborative program, in which the inhabitants of the community participate, gave legitimacy to the actions carried out.
\end{abstract}

Key words: Tourism Management, Endogenous Resources, Cultural Heritage, Central Mexico.

\section{RESUMEN}

En la presente investigación se describe la aplicación de una estrategia de gestión turística de los recursos patrimoniales en el poblado de San Francisco Oxtotilpan, Estado de México. Se trata de una comunidad matlatzinca que cuenta con recursos naturales enmarcados en una vasta riqueza cultural, que se han mantenido a través de la preservación de sus costumbres y tradiciones. Se desarrolló un estudio de caso, con un enfoque centrado en la metodología de investigaciónacción, con el objetivo de identificar los procesos de gestión patrimonial participativa en la implementación de estrategias de turismo rural. La aplicación de un programa colaborativo, protagonizado por los habitantes de la comunidad otorgó legitimidad a las acciones desarrolladas.

Palabras clave: Gestión Turística, Recursos Endógenos, Patrimonio Cultural, Centro de México.

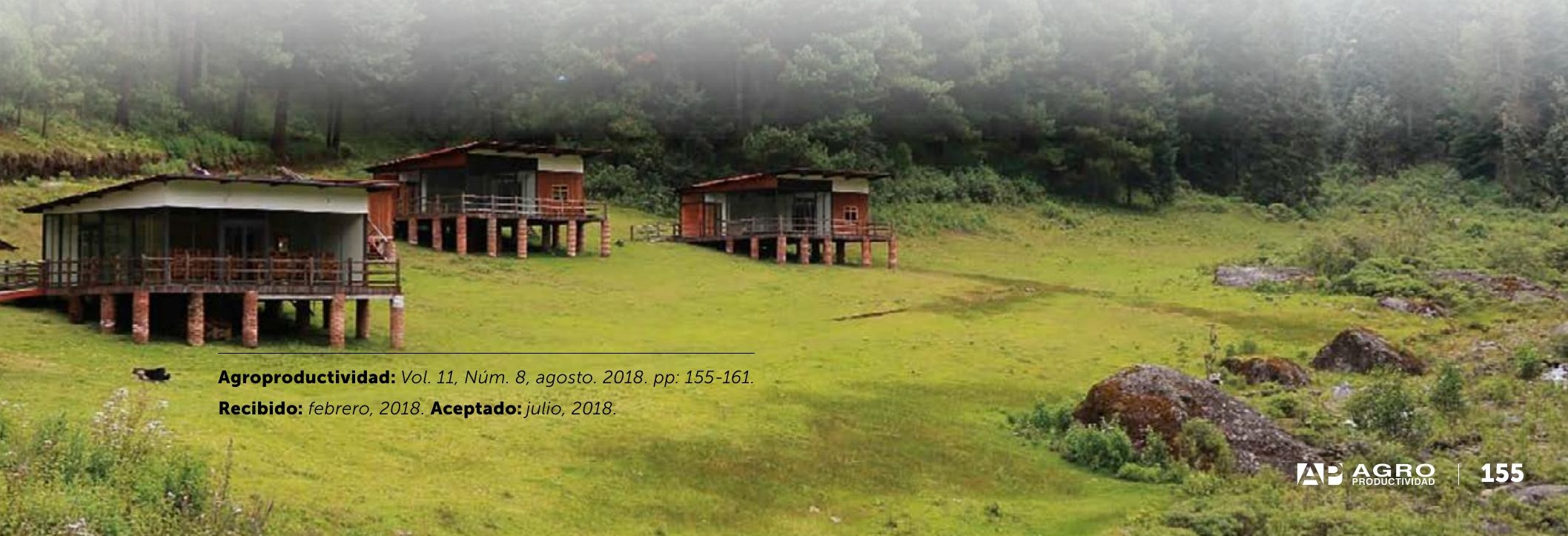




\section{INTRODUCCIÓN}

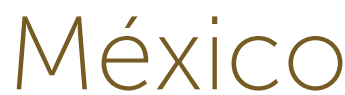

es un país culturalmente diverso,

los grupos étnicos que habitan en su territorio mantienen la esencia de sus costumbres y tradiciones, cumpliendo importantes funciones en la preservación del patrimonio cultural. Los pueblos indígenas son aquellos que descienden de poblaciones que habitaban en el territorio nacional al iniciarse la colonización y que conservan sus propias instituciones sociales, económicas, culturales y políticas, o parte de ellas (Constitución Política de los Estados Unidos Mexicanos, Art. 2ㅇ)

\section{Una aproximación al concepto de patrimonio}

La UNESCO (2003), define al patrimonio como el conjunto de conocimientos, significados, símbolos, costumbres, tradiciones y formas de relacionarse para entender el mundo y vivir la cotidianeidad de un grupo social específico, en un ámbito físico y temporal determinado.

El patrimonio lo constituyen bienes tangibles e intangibles que tienen un alto contenido simbólico, lo que les hace merecedores de una especial protección, no sólo relacionada con su conservación, sino también con el uso que se pueda hacer de ellos (Ballart y Tresserras, 2001; Hernández, 2002; Velasco, 2009).

El patrimonio cultural es todo aquello que socialmente se considera digno de ser preservado (Prats, 2005); e incluye la herencia de cada pueblo como pueden ser: sitios arqueológicos, expresiones arquitectónicas y cultura material, además de los bienes contemporáneos, visibles e invisibles (García-Canclini, 1999; Ciselli, 2015).

\section{Gestión turística del patrimonio cultural}

La gestión turística del patrimonio cultural es la aplicación de conocimientos específicos para la adecuación de los bienes culturales como recursos turísticos, orientada a la conservación de los mismos. Derivado de lo anterior, se presenta el hecho paradójico de que el patrimonio experimenta una creciente presión, económica y política, relacionada con sus usos turísticos, evidente a partir del progresivo aumento de la oferta de turismo cultural (Velasco, 2009). Dados los riesgos que el turismo representa para el patrimonio, la adecuada gestión turística debe ser un medio de conservación y difusión que permita la continuidad de los recursos endógenos de las comunidades campesinas (ThoméOrtiz, 2008).
Para Bermúdez, Arbeloa y Giralt (2004) la gestión patrimonial implica la actuación integral, orientada a optimizar unos recursos y obtener rendimiento de los mismos. El gestor cultural, frente a los recursos patrimoniales, desempeña una labor de manejo estratégico, cuya meta final es su rentabilidad en términos sociales, económicos y financieros, reiterando que dicho actor debe buscar siempre el beneficio social sobre los intereses particulares.

En este sentido, como lo menciona Velasco (2009), el verdadero valor del patrimonio es que sea reconocido como valioso por los miembros de la sociedad en la que éste está inserto. Ello permite que los bienes mantengan su capacidad simbólica y trasmitan los valores de la colectividad a la que representan, aunque se haya modificado el uso original de los mismos.

Por tanto, el auge del turismo y la diversificación en las actividades de los turistas se convierten en una oportunidad para el patrimonio cultural, diseminado por un territorio que empieza a ser percibido como destino turístico. Simultáneamente, hay que considerar que la masificación, la falta de autenticidad y la resistencia a reinvertir parte de los beneficios económicos obtenidos por el turismo en los bienes de patrimonio, aparecen como amenazas graves para los mismos. (Velasco, 2009).

En este sentido, la gestión es un proceso orientado a responder a las necesidades específicas de un territorio, proyectando la transformación de la realidad, a través del cambio social, cultural o económico de un colectivo determinado; gestionar implica reaccionar dinámicamente a los constantes cambios y movimientos, tomar las mejores decisiones para el desarrollo de proyectos (CNCA, 2009).

De acuerdo con Querol (2010) las acciones necesarias para la gestión del patrimonio cultural son: conocer, planificar, controlar y difundir el conjunto de bienes patrimoniales. Por su parte, la Secretaría de Estado para Asuntos Económicos del Perú, plantea que la gestión de un destino turístico varía de acuerdo a su situación y necesidades particulares, siempre que sean considerados tres aspectos básicos: i) Planificación y desarrollo del destino, ii) Desarrollo de productos y servicios, iii) Promoción y comunicación del destino turístico (SECO, 2014).

La gestión del Patrimonio Cultural debe tener una cadena lógica de intervención como lo exponen Bermúdez et al. (2004) quienes mencionan que la intervención 
en el patrimonio es una secuencia encadenada de acciones, integrada por cuatro niveles relacionados y dependientes: investigación, protección, conservación y restauración, difusión y didáctica. El objetivo de esta investigación es identificar los procesos de gestión patrimonial participativa en la implementación de estrategias de turismo rural en una comunidad indígena del Altiplano Central Mexicano.

\section{MATERIALES Y MÉTODOS}

La investigación se desarrolló a través de un estudio de caso de tipo cualitativo (Stake, 2013) recuperando elementos de la metodología de investigación-acción (Ander Egg 2003) la cual se enfoca en los grupos de población, que pasan de ser "objeto" de estudio a "sujeto" protagonista de la investigación, a partir de una estrecha vinculación entre el investigador y la comunidad a estudiar (Alberich, 2006). La fase inicial del diseño metodológico contempló el desarrollo de un diagnóstico participativo (Verdejo, 2003) para identificar la problemática específica de la zona de estudio (Figura 1).

Un aspecto fundamental fue el acompañamiento de la comunidad para la gestión del patrimonio a partir del turismo rural en San Francisco Oxtotilpan, para tal efecto dando continuidad a la metodología para la gestión del patrimonio planteada por Bermúdez et al. (2004), se desarrollaron los siguientes pasos: identificación, clasificación y diagnóstico del patrimonio para el diseño de un producto turístico y la aplicación de la prueba piloto, mediante el modelo de laboratorio social de turismo experiencial (Thomé-Ortiz, 2016; López-Mathamba, Peña-Levano, Thomé-Ortiz y Escalante, 2017).

\section{RESULTADOS Y DISCUSIÓN}

El comisariado ejidal de San Francisco Oxtotitlpan, ini- ció la gestión del apoyo económico para la construcción de dos cabañas y un restaurante, que conforman el Parque Ecoturístico Matawi, a través del Programa de Turismo Alternativo en Zonas Indígenas (PTAZI) de la Comisión Nacional para el Desarrollo de los Pueblos Indígenas (CDI) (Zarazúa, 2014). En la mayoría de las comunidades rurales la infraestructura turística, obtenida mediante estos programas, es subutilizada (GonzálezDomínguez; Thomé-Ortiz y Osorio-González, 2018) puesto que los actores locales, principalmente campesinos, no conocen el manejo de la actividad turística, les es complicado el diseño de productos turísticos, su operatividad, así como el desarrollo de estrategias para la promoción y comercialización de su oferta turística (Figura 2).

Con base en lo anterior, un aspecto clave fue el acompañamiento a la comunidad para la gestión del turismo rural, con lo que fue posible apoyar al grupo inicial de prestadores de servicios turísticos para la capacitación

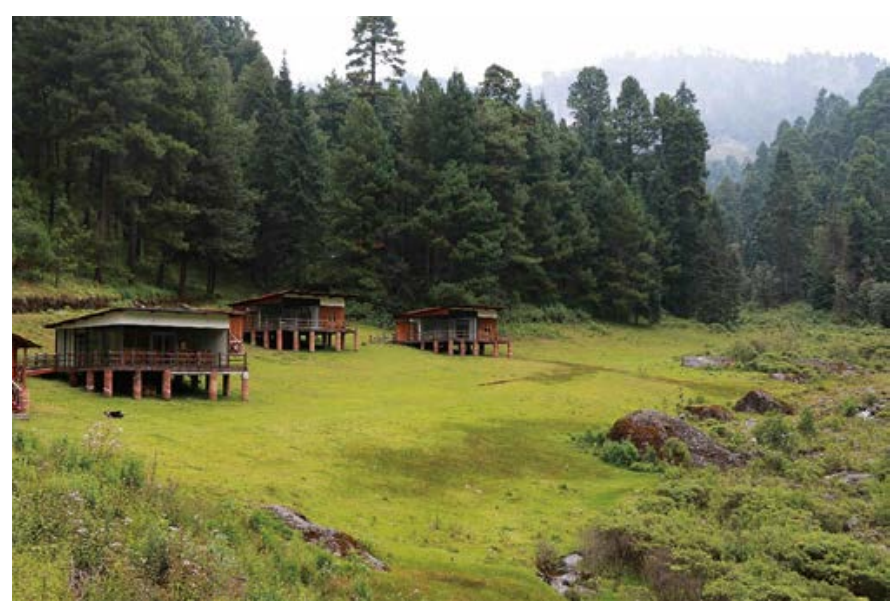

Figura 2. Parque Ecoturístico Matawi, San Francisco Oxtotilpan, Estado de México. Autor: Jonathan Aguirre Zuñiga.

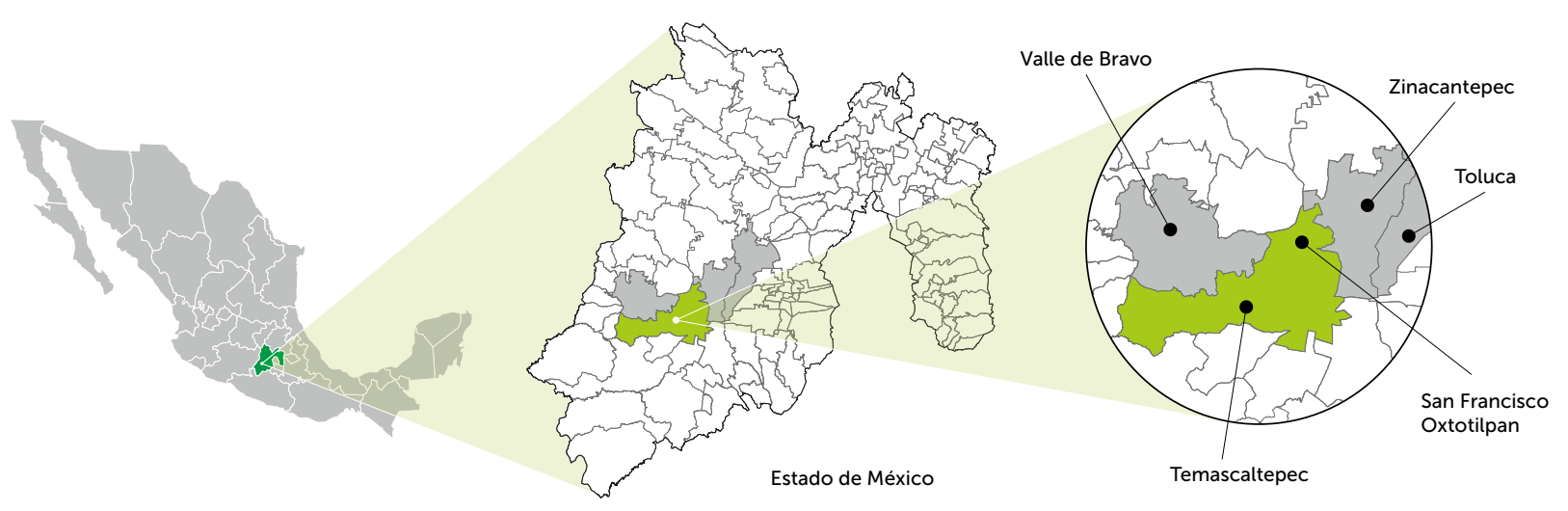

Figura 1. Ubicación de San Francisco Oxtotilpan, en el Estado de México. Fuente: Jiménez-Ruiz, Thomé-Ortiz y Burrola-Aguilar (2016). 
turística, la autoevaluación de los conocimientos adquiridos y la experiencia vivencial en la prestación de servicios turísticos.

El desarrollo de la propuesta inició con un diagnóstico del potencial turistico del territorio (Zimmer y Grassmann, 1996), a través de investigación en campo y documental, con el propósito de conocer los recursos específicos que integran el patrimonio cultural de la comunidad, así como ubicar los conocimientos, disposición de los actores locales a participar, experiencia de la comunidad y sus necesidades de capacitación.

\section{Patrimonio cultural matlatzinca}

En la comunidad de San Francisco Oxtotilpan, se encuentra una vasta riqueza cultural intangible, ya que algunos de sus habitantes, aún conservan la lengua matlatzinca, celebran ritos como el de realizar una visita al volcán para solicitar a sus deidades la lluvia, consumen alimentos y bebidas tradicionales como el charapi (licor de zarza), maices nativos, hongos silvestres y quelites, realizan el ritual del baño prehispánico (temazcal) y conservan costumbres y tradiciones.

\section{a) Lengua matlatzinca}

La lengua matlatzinca está considerada dentro de la rama otomangue otomípame. En ésta hay cuatro familias: pame, jonaz, matlatzinca y otomazahua. En la actualidad dicha lengua está desapareciendo debido a que, cada vez, son menos las personas que la hablan. La mayoría de la población de San Francisco es bilingüe pero existe un acusado uso del español y una reducción en el empleo de la lengua autóctona.

\section{b) Indumentaria}

La vestimenta tradicional de los matlatzincas se caracteriza, en el caso de los hombres, por el uso de un calzón de manta blanca que llega a los tobillos y camisa del mismo material, gabán de lana liso, huarache y sombrero de palma, además de una faja bordada en color rojo que sirve para sostener el calzón. En el caso de la mujer, una falda larga de lana, llamada enredo o chincuete, blusa floreada y faja (techiti) para sostener la falda. Actualmente, la vestimenta tradicional se ha sustituido por ropa de confección industrial, pues los atuendos típicos se usan, exclusivamente, en las festividades de este pueblo, por lo que la Comisión Nacional para el Desarrollo de los Pueblos Indígenas (CDI) apoyó a un grupo de mujeres de la comunidad para la realización de un taller de rescate de indumentaria.

\section{c) Gastronomía}

De la gastronomía es importante mencionar el pulque (totapi), el cual tiene un alto consumo en la comunidad, tiene un saber hacer tradicional, que ha sido heredado desde la época prehispánica. Actualmente lo producen sólo seis familias y es utilizado para autoconsumo.

En cuanto a los alimentos típicos se encuentran el tamal de ceniza, de frijoles o de haba, los hongos silvestres, el jocoque (leche cortada), el chilacayote con pipian (chimu), chivatitos (quelites), coquelites, atole y las tortillas de trigo con maíz.

\section{d) Fiestas patronales}

Las fiestas patronales más importantes se relacionan con la religión católica pero tienen también un vínculo con el ciclo agrícola y con el culto al volcán Xinantécatl (Nevado de Toluca), que consideran la representación de Dios en la Tierra (Granados, 2011). La fiesta más importante es la del santo patrono San Francisco de Asís, realizada el 4 de octubre, el festejo dura aproximadamente una semana, participan con una cooperación casi todas las familias del pueblo.

Otra fiesta relevante es el Día de Muertos, en la cual se coloca una ofrenda en forma de arco, en la que se cuelga pan sobre un petate con flores de cempaxúchitl, donde se ofrece mole, pulque y copal a los difuntos. Una tradición en esta fecha, es que si a algún muchacho le gusta una joven soltera de la casa anfitriona, éste le avienta un chilacayote cerca de los pies, si al padre de la joven le simpatiza el muchacho, lo invita a tomar pulque.

El 15 de mayo se festeja a San Isidro Labrador, se realiza una misa, procesión y la tradición de la ropa vieja, en la que hombres se visten de mujeres y bailan al centro de la plaza, también se adornan arados y se realiza una procesión con la imagen del Santo.

\section{e) Rituales}

Con la finalidad de pedir que lleguen las lluvias, se realiza una ceremonia en el cráter del volcán Xinantécatl, considerado para los matlatzincas la "morada de los dioses". Dicho ritual inicia con una caminata desde el centro de la comunidad hasta el volcán, donde pernoctan y realizan oraciones en el cráter; ahí se ingieren, durante la noche, bebidas 
alcohólicas que tienen un fin ritual. Esta ceremonia tiene lugar en el mes de mayo y se mantiene desde la época prehispánica. Al regreso se realiza un banquete que es ofrecido por los mbechoques o fiscales de la iglesia principal (Granados, 2011).

Otra práctica ritual, relacionada con la fertilidad de la tierra, consiste en preparar un tamal de maíz cocido con cenizas de madera en lugar de cal, el cual es enterrado en la casa y rodeado por cuatro veladoras que representan los puntos cardinales; en palabras de los habitantes: "...alimentamos la tierra para que ella nos alimente a nosotros...". (Granados 2011)

\section{f) Danzas}

Existen danzas como la "de los Pastores", que se escenifica el 24 de diciembre para celebrar el nacimiento de Cristo y la de los negritos, que se lleva a cabo el 19 de marzo en el festejo del Quinto Sol, donde el "Jefe Supremo" de la etnia realiza un ritual de agradecimiento a los cuatro puntos cardinales.

\section{Aplicación de programa de Gestión Turística del patrimonio cultural en la comunidad de San Francisco Oxtotilpan}

Paso 1. Conformación de grupo de trabajo

Este grupo se conformó a partir de una convocatoria abierta realizada en la comunidad, en el contexto de la asamblea de ejidatarios, en la cual se decidió que podían participar en la prestación de los servicios turísticos quienes asistieran a las reuniones para tal efecto y tomaran las capacitaciones pertinentes. El grupo inicial estaba conformado por 20 personas (11 mujeres y 9 hombres), cuyas edades oscilaban entre 15 y 60 años. El comisariado ejidal designó a uno de sus integrantes como el encargado del parque ecoturístico Matawi y la convocatoria se encuentra permanentemente abierta para quienes deseen integrarse al proyecto.

12 personas tienen edades que oscilan entre 15 y 20 años, 6 personas entre 21 y 40 años y una señora de 60 años, la mayoría son familiares de algún ejidatario.

La aplicación de la primera prueba piloto se llevó a cabo en el mes de septiembre de 2014, (los días 12 y 19), la organización de la actividad, se realizó con el acompañamiento de académicos de la Universidad Autónoma del Estado de México, que previamente realizaron un diagnóstico.
Con la finalidad de difundir el patrimonio cultural matlatzinca y responder a las necesidades contemporáneas de ocio y recreación, se programaron actividades que permitieran compartir rasgos de su indumentaria, lengua, gastronomía, costumbres y tradiciones.

Paso 2. Diseño de propuesta turística.

A continuación se describen e ilustran las actividades que conforman la propuesta turistica que fue construida, entre académicos y la comunidad, desde un enfoque participativo

\section{a) Recibimiento del grupo}

Habitantes de la comunidad, dan la bienvenida a los visitantes en lengua matlatzinca y utilizan la indumentaria tradicional. Posteriormente, se ofrece un desayuno que consiste en atole de trigo y pan de miztu (gato) como se conoce en la comunidad, el cual es elaborado con harina de maíz. Una vez concluido el desayuno, se procede a la división del grupo en equipos, asignándole 15 personas a cada guía con lo que se respeta la capacidad de carga para cada sendero definida en estudios previos (López-Mathamba, Peña-Levano, Thomé-Ortiz y Escalante, 2017). A cada equipo se la asigna el nombre de un elemento natural en matlatzinca: jani (tierra), tawi (agua) y nijnupi (aire). Enseguida, se da inicio a la caminata hacia la parte alta del cerro, donde se encuentran las cabañas y el restaurante. Durante el recorrido, de aproximadamente 45 minutos, los visitantes pueden identificar los recursos naturales y observar las características físico-geográficas del lugar. Los guías responden las preguntas de diversa índole, formuladas por los visitantes.

\section{b) Taller de Techiti}

Los integrantes del Grupo Piloto, ataviados con la indumentaria matlatzinca, explican el significado de la indumentaria y de las acciones que se están realizando para su rescate. Invitan a los visitantes a utilizar el telar para conformar prendas de lana y explican cómo tiñen la lana con base en productos naturales. En este taller se comercializan las artesanías de lana elaboradas por un grupo de mujeres, con lo cual se obtienen ingresos adicionales (Figura 3).

\section{c) Plática de la cosmovisión matlatzinca}

En ésta plática se explican las costumbres y tradiciones ya mencionadas en el apartado del patrimonio cultural matlatzinca, tales como las festividades de Día de 
Muertos, el cambio de mbechoques, el ritual en el volcán, las fiestas patronales y las leyendas.

\section{d) Taller Gastronómico}

En éste taller se muestran los productos típicos de la región: hongos silvestres, quelites y maíz nativo, así como algunos de sus platos más representativos. Los visitantes preparan platillos típicos como: tamales de ceni-

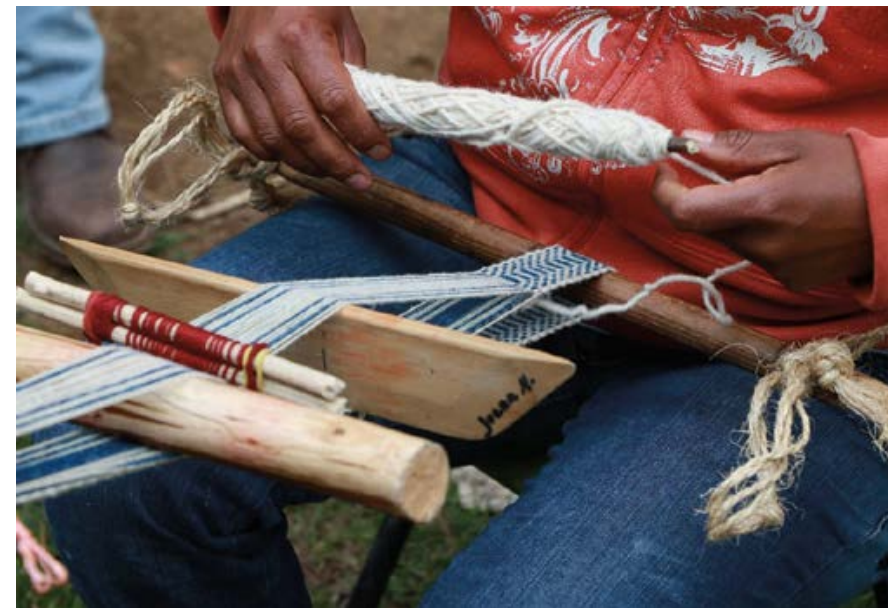

Figura 3. Taller de Techiti. Autor: Jonathan Aguirre Zuñiga. haciéndolos participes de las mismas (Figura 4).

\section{g) Temascal}

Posteriormente, se regresa al centro de la comunidad donde se visitan casas que tienen temascal (baño de vapor indígena), en las cuales cada uno de los grupos participa de éste ritual. Dentro del temascal, a las personas se les proporcionan hierbas para "varearse", es decir za y de frijol, chilacayote en pipián, quelites, chivatitos, hongos, elotes cocidos, tortillas hechas a mano y licor de zarza. Finalmente se realiza una degustación de los productos.

Al final del recorrido se lleva a cabo el raspado artesanal de maguey para la obtención de pulque y se explica la elaboración de curados.

\section{e) Comida}

El menú se diseñó a partir de los alimentos típicos más representativos de la comunidad, tales como: sopa de haba, chilacayote en pipián, quelites y agua zarza.

Al final de la comida se realiza un ejercicio de interacción entre los integrantes del grupo piloto y los visitantes, en la cual se intercambian ideas acerca de su emprendimiento turístico, expresando su opinión sobre la actividad.

\section{f) Visita a Invernadero}

El invernadero fue gestionado por el grupo de ejidatarios, quienes recibieron apoyo de la Comisión Nacional Forestal (CONAFOR) y actualmente venden árboles para la reforestación de las comunidades vecinas. En éste espacio, se muestra a los visitantes las actividades cotidianas como el sembrado, embolsado y trasplante de árboles,

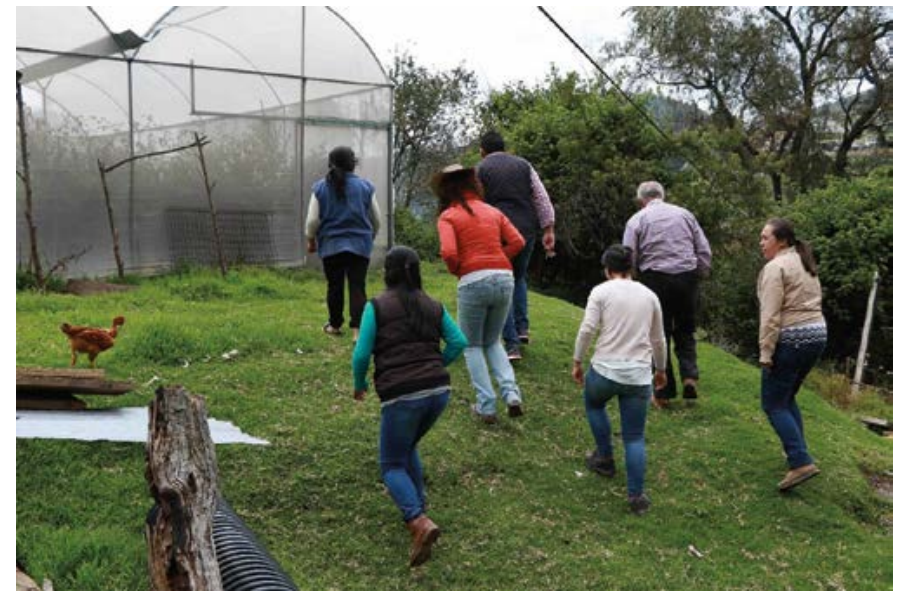

Figura 4. Visita a invernaderos. Autor: Jonathan Aguirre Zuñiga golpearse suavemente con las mismas con la finalidad de vigorizarse, al terminar se enjuagan con una infusión herbal para completar el ritual.

Las actividades desarrolladas en la prueba piloto, han sido modificadas en visitas posteriores, dependiendo de la temporalidad y de los recursos existentes, por ejemplo, se ha incluido la visita a un criadero de caracol, a diversos invernaderos (fresa, chile, nopal), se realiza un taller de elaboración de un producto (gel, jabón o shampoo) a base de fresa y se han desarrollado algunos recorridos de turismo micológico para pequeños grupos (Thomé-Ortiz, 2015).

\section{CONCLUSIONES}

a gestión del patrimonio cultural de la comunidad de San Francisco Oxtotilpan, implica la construcción participativa de una propuesta turística, basada en las características específicas de la etnia matlatzinca, con lo que es posible ofertar una experiencia recreativa diferenciada, fuertemente ligada al territorio y con un sello de identidad local. La prueba piloto permitió a los integrantes del grupo, prepararse operativamente, autoevaluar su desempeño, vivir la experiencia de otorgar un servicio turístico y detectar sus debilidades, otorgando con ello herramientas prácticas para los actores locales interesados en 
convertirse en prestadores de servicios, para insertarse de manera consciente y paulatina al desarrollo de la actividad turística.

El planteamiento del turismo rural en San Francisco Oxtotilpan, se gestó a partir de la academia de manera colaborativa. Sin embargo, este ejercicio presenta la limitación de ser un estudio de caso particular, bajo la perspectiva de la comunidad, por lo que sus alcances únicamente pueden ser válidos para la unidad de análisis estudiada y simplemente suponen un referente para otros casos que presenten características similares. Pese a lo anterior, los resultados obtenidos son de utilidad para los actores locales implicados en la actividad turística, en términos de la gestión de los recursos locales. Futuras investigaciones deberán integrar la perspectiva del mercado turístico y de las instituciones públicas impulsoras del turismo indígena, así como realizar comparaciones con casos que presenten características similares en otros contextos del país y del mundo.

\section{LITERATURA CITADA}

Alberich N.T. 2006. La Agenda 21 de la Cultura. Un instrumento para el desarrollo. Castellón de la Plana, España. Extensión Universitaria. Universidad Jaume I. Castellón

Ander-Egg E. 2003. Repensando la investigación-acción-participativa Madrid, España.Ed. Lumen-Humanitas.

Ballart J., Tresserras J. 2001. Gestión del patrimonio cultural. Barcelona, España. Ed. Ariel.

Bermúdez A., Arbeloa J. V., Giralt A. 2004. Intervención en el patrimonio cultural: creación y gestión de proyectos. Madrid, España. Ed. Sintesis.

Ciselli G. 2015. El Patrimonio Cultural: entre la identidad y el ambiente. Revista electrónica de Patrimonio Histórico, 9: 4-22.

Constitución Política de los Estados Unidos Mexicanos Art. 2․ Obtenido de: https://mexico.justia.com/federales/constitucion-politicade-los-estados-unidos-mexicanos/

Consejo Nacional de la Cultura y las Artes (CNCA). 2009. Guía para la gestión de proyectos culturales .Consejo Nacional de la Cultura y las Artes de Chile. Obtenido de: https://issuu.com/cultudocs/ docs/guia_para_la_gestion_de_proyectos_culturales/75

García-Canclini N. 1999. Los usos sociales del patrimonio cultural. Cuadernos, 16-33

González-Dominguez I., Thomé-Ortiz H., Osorio-González R. 2018. Políticas turísticas y etnoturismo: entre la rururbanización y el desarrollo de capacidades. PASOS. Revista de Turismo y Patrimonio Cultural, 16: 21-36

Granados R., Pérez C. 2011. Alimentación tradicional y adaptación al mercado en San Francisco Oxtotilpan, México. Culinaria Revista virtual especializada en gastronomía, 51-75

Hernández F. 2002. El Patrimonio Cultural: la memoria recuperada. Madrid, España. Ed. Trea.

Prats L. 2005. Concepto y gestión del patrimonio local. Cuadernos de Antropología social. 21: 17-35

Jiménez-Ruiz A., Thomé-Ortiz H., Burrola-Aguilar C. 2016. Patrimonio biocultural, turismo micológico y etnoconocimiento. El periplo sustentable. 30: 180-205.

López-Mathamba L., Peña-Levano L., Thomé-Ortiz H. Escalante C. 2017. Matlatzinca Interpretative Path and Food Landscape (MIP) Social Touristic Project. Journal of the ASFMRA. 152-166.

Querol M. A. 2010. Manual de Gestión del Patrimonio Cultural. Madrid España. Ed. Akal.

Secretaría de Asuntos Económicos (SECO). 2014. Conceptos básicos para la gestión de destinos turísticos. Destinos del Perú. Obtenido de: https://www.swisscontact.org/fileadmin/user_upload/ COUNTRIES/Peru/Documents/Publications/Conceptos_ basicos_para_la_gestion_de_destinos_turisticos.pdf.

Stake R. E. 2013. Multiple case study analysis. New York, United States of America. Guilford Press.

Thomé-Ortiz H. 2008. Turismo rural y campesinado, una aproximación social desde la ecología, la cultura y la economía. Convergencia. 15 (47): 237-261

Thomé-Ortiz H. 2015. Turismo micológico. Una nueva mirada al bosque. Ciencia y Desarrollo. 14-19.

Thomé-Ortiz, H. 2016. Turismo Rural y Sustentabilidad. El caso del turismo micológico en el Estado de México. En: CarreñoMelendez, F. y Vázquez-González, Y. Ambiente y Patrimonio Cultural. Pp.: 43-69. Toluca, México. CEDES-UAEMEX

United Nations Educational, Scientific and Cultural Organization (UNESCO). 2003. Convención para la salvaguardia del patrimonio cultural inmaterial. Paris.

Velasco G.M. 2009. Gestión turística del patrimonio cultural: enfoques para un desarrollo sostenible del turismo cultural. Cuadernos de turismo. 23: 237-254

Verdejo M.E. 2003. Diagnóstico rural participativo: guía práctica. Santo Domingo, República Dominicana. Ed. Centro Cultural Poveda.

Zarazúa J.A., Mazabel D., Camacho-Bernal M.T., Trench T. 2014 Política pública y turismo en México: análisis exploratorio del programa de turismo alternativo en zonas indígenas (PTAZI) en el ejido la fortuna del gallo giro, municipio de Las Margaritas Chiapas México. TURyDES. 7:2-21

Zimmer P., Grassmann, S. 1996. Evaluar el potencial turístico de un territorio. Madrid, España. Observatorio europeo LEADER. 